The Egyptian Journal of Hospital Medicine (April 2019) Vol. 75 (3), Page 2353-2357

\title{
aIncisional Negative Pressure Wound Therapy (INPWT) for Coronary Artery Bypass Grafting Patients
}

\author{
Ahmed M. El Ashkar, Ahmed N. Khallaf \\ Cardiothoracic Surgery, * Beni-suef University, Beni-suef, ** Fayoum University, Fayoum, Egypt \\ Corresponding author: Ahmed El Ashkar, Phone: 01222149535, E-mail: aelashkar@gmail.com
}

\begin{abstract}
Background: Surgical site infections are major complications of coronary artery bypass grafting using bilateral internal thoracic arteries. In this study we reviewed the results of using INPWT for high risk patients with coronary artery bypass grafting $(\mathrm{CABG})$ using bilateral internal mammary arteries.

Patients and methods: From January 2013 to December 2016, patients with coronary artery bypass grafting using bilateral mammary arteries were enrolled in this study. Patients were from Kasr El-Einy, Beni-Suef and Fayoum University Hospitals. The total number was 427 patients, where INPWT was used in 161 patient, and conventional sterilized dressing was done in 266 patients. 2 groups were matched for statistical analysis, the first group was the control and the second group was where INPWT was used. Results: The results related to sternal wound infections were similarly attributed to the conventional group (10.9\%) and the INPWT group $(10.2 \%)(\mathrm{P}=1.00)$. Patients treated with INPWT had less rate of infection than those with conventional dressing (5.5\% versus $10.2 \%, \mathrm{P}=0.210)$, this difference was not statistically significant. Interaction tests also showed comparable results for SSI (surgical site infections) among patients with or without significant co-morbidities. Conclusion: This study suggests that the use of INPWT did not decrease the incidence of sternal wound infections in patients who had CABG surgery using bilateral internal mammary harvesting (BIMA). A larger randomized study is needed to evaluate the efficacy of this technique. Keywords: Coronary artery bypass grafting; incisional negative pressure wound therapy; mediastinitis.
\end{abstract}

\section{INTRODUCTION}

Surgical site infections (SSIs) are fatal complications of open heart surgeries. SSIs are the Achilles heel of coronary artery bypass grafting using bilateral internal mammary arteries (BIMA) ${ }^{(1)}$. This is probably due to the impaired vascularity following the harvesting of both mammary arteries ${ }^{(2)}$. Although using BIMA has improved the long-term results of coronary artery bypass grafting $(\mathrm{CABG}){ }^{(3)}$, the risk of surgical site infection remains a limiting factor for its wide use as a method of coronary revascularization (4). Thus, several pre-, intra- and postoperative strategies have been adopted to decrease the risk of SSIs following BIMA use ${ }^{(5)}$. Recently, negative suction dressings for closed sternotomy wounds have been used with promising results to decrease the risk of infection ${ }^{(6)}$. The mechanism depends on decreasing the fluids and the infecting materials at the incision. In this study, the efficiency of this method with CABG patients using BIMA has been evaluated.

\section{PATIENTS AND METHODS}

427 patients with isolated CABG using BIMA were operated in Kasr El-Einy, Beni-Suef and Fayoum University Hospitals from January 2013 to December 2016. Antibiotic prophylaxis was done by cefazoline $1.5 \mathrm{gm}$ prior to the induction of anesthesia, with intraoperative redosing after 2 hours. Postoperatively, $1.5 \mathrm{gm}$ of cefazoline every 12 hours was given for 72 hours. The incision site was sterilized just before skin incision using povidone-iodine 10\%. BIMA harvesting was done in a skeletonized manner. The sternum was closed by 4 figure-of- 8 stainless steel sutures. The subcutaneous tissues were closed using 1-0 or 2-0 vicryl sutures. Skin closure was done using $3 / 0$ continuous subcuticular Monocryl sutures. Tight glycemic control was achieved using insulin intravenous infusion intraand post- operatively. Throughout the study period, INPWT was used as a preventive method over closed wounds in 161 patients. The remaining patients were dressed in a regular way. The prevalence of this technique was $9.1 \%$ in $2013,24.1 \%$ in $2014,51.7 \%$ in 2015 and $42.9 \%$ in 2016 ( $\mathrm{p}<0.001$ ). In the INPWT group, dressing was done immediately after skin closure and sterilization, in the operating room and under complete aseptic conditions. The suction pump was connected and adjusted to a negative pressure of $125 \mathrm{mmHg}$. The negative suction dressing was removed after 5-7 days. In the control group, a regular dressing was done at the operation room, and was changed the following day then regularly changed according to wound inspection every two days.

The criteria of assessment of this study was mainly the development of surgical site infection (SSI) of any degree, whether superficial, deep or mediastinitis. Those presenting with superficial SSI were only treated with antibiotics and wound care. Those with deeper infections required longer duration of antibiotic therapy, mostly I.V with reoperation for wound debridement and reclosure in cases of mediastinitis. The 
Incisional Negative Pressure Wound Therapy...

severity of surgical site infection was based on the operative site infection classification of the Center for Disease Control and Prevention (7). The additional results of this study were the in-hospital mortality, hospital stay, ICU stay, need for the Alice's, need for IABP, blood transfusion and reopening for bleeding. This study was approved by the ethical committee of each of the three institutions.

\section{Statistical analysis}

Statistical analysis was done using the logistic SAS, version 9.2 and the logistic SPSS version 24.0. Qualitative data were expressed as frequency and percentage and were compared by McNemar's test, Fisher test, and chi-squared tests. Quantitative data were expressed as mean \pm standard deviation (SD). Comparison of means was performed by the t-test. Propensity score was used to select two groups of patients, using INPWT or conventional dressing respectively, with similar basic criteria. The propensity score was estimated using non-parsimonious logistic regression method including the following variables: Age, gender, body mass index (BMI), diabetes, ejection fraction (LVEF) and EuroSCORE II ${ }^{(8)}$.

Propensity score matching was utilized using the nearest neighbor method, and a logistic propensity score with 0.2 diversion. In order to prove that interaction with other risk factors, interaction tests were done. ANOVA (analysis of variance), qualitative and quantitative interaction tests were used. These tests were done by relating sub-groups with relevant comorbidities, weather for all infections, superficial, deep infections or mediastinitis. $P$ values less than 0.05 were considered statistically significant ${ }^{(9)}$.

\section{RESULTS}

In this study, 38 patients had SSI (8.9\%), wether superficial (11 patients, 2.6\%) or deep or mediastinitis (27 patients, $6.3 \%$ ).

Table (1): Patients characteristics

\begin{tabular}{|c|c|c|c|}
\hline & Regular dressing $(\mathrm{n}=266)$ & Negative suction $(\mathrm{n}=161)$ & P-value \\
\hline Age (years) & $66.3 \pm 10.0$ & $67.6 \pm 8.7$ & 0.162 \\
\hline Females & $28(10.5)$ & $26(16.2)$ & 0.090 \\
\hline BMI (kg/m2) & $27.7 \pm 4.0$ & $29.5 \pm 5.4$ & $<0.001$ \\
\hline Diabetes & $73(27.4)$ & $106(65.8)$ & $<0.001$ \\
\hline LVEF 30-50\% & $78(29.3)$ & $45(27.9)$ & 0.929 \\
$<30 \%$ & $16(6.0)$ & $2.9 \pm 0.8$ & 0.297 \\
\hline umber of distal anastomoses & $2.8 \pm 0.7$ & $62.6 \pm 21.8$ & 0.938 \\
\hline Cross clamp time (min) & $62.4 \pm 20.7$ & $94.9 \pm 21.8$ & 0.938 \\
\hline CPB time (min) & $95.0 \pm 26.5$ & $3.2 \pm 1.3$ & 0.013 \\
\hline EuroSCORE II (\%) & $2.9 \pm 1.4$ & & \\
\hline
\end{tabular}

Table (2): Culture and sensitivity results

\begin{tabular}{|c|c|}
\hline Organism & Number of patients \\
\hline Staph aureus & 11 \\
\hline Staph epidermidis & 9 \\
\hline Staff coagulase negative & 9 \\
\hline E. coli & 4 \\
\hline Seratia Marcescens & 2 \\
\hline Propionibacterium anes & 2 \\
\hline Staph scleiferi & 1 \\
\hline Proteus Mirabilis & 1 \\
\hline Morganella Morganii & 1 \\
\hline Klebsiella pneumoniae & 1 \\
\hline Enterobacter cloacae & 1 \\
\hline Enterobacter aerogenes & 1 \\
\hline Achromobacter & 1 \\
\hline Gram +ve bacteria & 1 \\
\hline Sterile culture & 1 \\
\hline
\end{tabular}

*more than one organism were found in 7 patients. 
Table 3: Complications

\begin{tabular}{|l|c|c|c|}
\hline & $\begin{array}{c}\text { Regular dressing } \\
(\mathrm{n}=266)\end{array}$ & $\begin{array}{c}\text { Negative suction } \\
(\mathrm{n}=161)\end{array}$ & P value \\
\hline All infections & $21(7.9)$ & $17(10.6)$ & 0.349 \\
\hline Superficial SSI & $4(1.5)$ & $7(4.3)$ & 0.111 \\
\hline Deep SSI & $5(1.9)$ & $3(1.9)$ & 1.000 \\
\hline Mediastinitis & $12(4.5)$ & $7(4.3)$ & 0.937 \\
\hline Dialysis & $6(2.3)$ & $4(2.5)$ & 1.000 \\
\hline Need for IABP & $11(4.1)$ & $2(1.2)$ & 0.144 \\
\hline Reopening for bleeding & $4(1.5)$ & $3(1.9)$ & 0.777 \\
\hline Blood transfusion & $161(60.5)$ & $102(63.49)$ & 0.560 \\
\hline ICU stay (days) & $4.7 \pm 3.0$ & $6.0 \pm 6.0$ & 0.004 \\
\hline Hospital stay (days) & $12.9 \pm 9.0$ & $13.8 \pm 11.5$ & 0.016 \\
\hline Hospital mortality & $7(2.6)$ & $3(1.9)$ & 0.749 \\
\hline
\end{tabular}

Despite the absence of statistically significant differences, we can note the decreased number of surgical site infection in the negative suction group (17 patients versus 21 patients in the conventional dressing group, $\mathrm{P}=0.349)$. Also, the in-hospital mortality was higher in the conventional group ( 7 versus 3 patients, $\mathrm{P}=$ 0.749). Otherwise, the incidence of superficial wound infection, was relatively higher in the negative suction group ( 7 versus $4, \mathrm{P}=0.111$ ), also the total hospital stay was higher than in the conventional group (13.8+-11.5 versus $12.0+-9.0, p=0.016$ ) (statistically significant).

During the period of study, there were no patients with superficial, deep wound infection or mediastinitis who required multiple reoperations. No mortality directly related to infections was noted. Meanwhile there were morbidities related to the prolonged duration of hospitalization, the side effects of medications (antibiotics) and the significant psychological effects on the patients. The pairing propensity score resulted in 128 pairs with similar basic characteristics. Meanwhile, the left ventricular ejection fraction was lower in the conventional dressing cohort. The EuroSCORE II values were higher in the negative suction group. Mc Nemar test showed no significant differences regarding all infections (10.9\% in the conventional group versus $10.2 \%$ is the negative suction group, $\mathrm{P}=1.00$ ).

Patients treated with negative suction dressing presented with less deep wound infection and mediastinitis than the control group 5.5\% versus $10.2 \%$ $\mathrm{P}=0.210$ ), however, the difference had no statistical significance. Interaction tests have shown that the results were coherent between the patients with or without significant comorbidities (Table 4).

The gender (male OR: 0.34 versus female OR: 2.96, $\mathrm{P}=0.934$ ), Diabetes (non- diabetics OR: 0.33 versus diabetics OR: $3.08, \mathrm{p}=0.684$ ), and BMI ( $\mathrm{BMl}<30$ OR: 0.87 versus $\mathrm{BMI}>30$ OR: $1.15, \mathrm{p}=0.177$ ) had no effect on the total number of infections.

Table 4: Interaction tests in subgroups

\begin{tabular}{|c|c|l|c|c|c|}
\hline & OR & $95 \%$ CI & Interaction & Chi $^{2}$ & p- value \\
\hline Regular dressing Vs. INPWT & 1.00 & 0.42 & 2.38 & & \\
\hline Male & 0.34 & 0.10 & 1.13 & 0.17 & 0.934 \\
Female & 2.96 & 0.89 & 9.87 & & \\
\hline Age <70 & 0.55 & 0.18 & 1.68 & 0.72 & 0.665 \\
Age >70 & 1.83 & 0.60 & 5.63 & & \\
\hline Non-diabetic & 0.33 & 0.09 & 1.23 & 0.04 & 0.684 \\
Diabetic & 3.08 & 0.81 & 11.61 & & 0.932 \\
\hline LVEF >50\% & 0.35 & 0.11 & 1.11 & 0.11 & 0.177 \\
LVEF <50\% & 2.86 & 0.90 & 9.10 & & \\
\hline BMI <30 & 0.87 & 0.28 & 2.67 & 0.20 & \\
BMI >30 & 1.15 & 0.37 & 3.54 & & \\
\hline
\end{tabular}

OR: Odds Ratio, CI: Confidence interval

LVEF: left ventricular ejection fraction

BMI: Body mass index 


\section{DISCUSSION}

Despite the initial encouraging reports of Atkins et al ${ }^{(10)}$ and Colli and Camara ${ }^{(11)}$, no significant benefit was noted with the use of INPWT. The results have shown that the use of INPWT has not significantly decrease the incidence of SSI in CABG patients with BIMA harvesting.

These results are clinically relevant, because these patients have a major risk of sternal wound complications, which hinders the wide use of BIMA harvesting ${ }^{(12)}$.

The long hospital stay in the INPWT group can be attributed to the multiple risk factors among this patient population (uncontrolled postoperative blood sugar, chronic obstructive pulmonary disease, and overweight), which requires close observation, hence a prolonged hospital stay. The duration needed to apply the INPWT dressing for 7 days has also contributed to the longer total hospital stay. An increase in the total number of superficial SSI was noted in that INPWT group, which can be attributed to that close accurate observation of these patients, as well as the multiple risk factors, which together might have caused a diagnostic bias.

Interaction analysis had not shown evident beneficial effect of INPWT in the patient subgroups with relevant clinical comorbidities. Although studies done by Grauhan and colleagues ${ }^{(\mathbf{1 3}, 14)}$ have shown decreased risk of SSI after adults cardiac surgery, yet one of the studies lacked the initial data for these patients, and the comparative analysis didn't correlate the initial and the operative covariables ${ }^{(13)}$. Another study including obese patients has shown that the risk of SSI was significantly lower with the use of INPWT when compared to conventional stride dressing $(4 \% \mathrm{Vs}$ $16 \%, \mathrm{P}=0.03)^{(14)}$.

This study has shown a decrease in the number of severe SSI, whether deep sternal wound infection or mediastinitis, with the use of INPWT when compared to conventional sterile dressing. Although this difference was statistically insignificant, a larger study with adequate study samples is required to prove the potential benefit of this technique for patients undergoing cardiac surgeries. In fact, to detect a 50\% decrease in incidence of SSI, whether deep or mediastinitis, the estimated sample size of matched cases should be 255 patients in each cohort. Thus, the post-hoc suggests that the present analysis is insufficient to detect a decrease in such a serious risk. Meanwhile, the randomization of INPWT and conventional sterile dressing for 510 patients undergoing BIMA harvesting is not realistic. A larger study population is needed to demonstrate a significant reduction in deep SSRI with the use of INPWT, when the total rate of $2.4 \%$ of this complication is considered (15) .Another important fact is the cost analysis which is also necessary to estimate the financial burden of a large number of patients to be treated using this method to prevent a possible SSI. The limited number of patients, and the suboptimal quality of the studies evaluating INPWT in cardiac surgery hinder conclusive results. The potential benefits of INPWT can be exported from the results of other randomized studies in other surgical fields. Two recent randomized studies have proven a significant advantage for INPWT after laparotomies' (16). Nevertheless, another larger randomized study done by Shen et al. ${ }^{(17)}$ didn't show any advantage using INPWT in abdominal surgery. Moreover, INPWT didn't reduce the risk of SSI in other randomized studies related to different surgical specialties ${ }^{(18,19)}$. These results suggest that the benefit of INPWT after surgeries is still controversial and its regular use cannot be recommended until accurate studies have proven its efficiency in reducing postoperative complications after cardiac surgery. The non-randomization is a major limiting factor of this study, still, data collection was prospective.

Data concerning SSI, culture and sensitivity, as well as medical and surgical management of the patients were detailed according to the predetermined criteria. Additionally, this study is not sufficient to detect a 50\% reduction of risk of SSI in the conventional sterile dressing cohort.

\section{CONCLUSION}

The results of this study suggest that the regular use of INPWT cannot significantly reduce the incidence of SSI in patients undergoing CABG using bilateral internal mammary grafting. Considering the previous reports showing the significant advantages of this method, we should ask if the power of this study was sufficient for such an uncommon procedure. A larger randomized study is clearly needed to accurately evaluate the efficiency of this technique for adult patients undergoing cardiac surgery.

\section{REFERENCES}

1. Kouchoukos NT, Wareing TH, Murphy SF, Pelate C, Marshall WG (1990): Risks of bilateral internal mammary artery bypass grafting. Ann Thorac Surg., 49: 210-217. 
2. Parish MA, Asai T, Grossi EA et al. (1992): The effects of different techniques of internal mammary artery harvesting on sternal blood flow. J Thorac Cardiovasc Surg., 104:1303-1307.

3. Lytle BW, Blackstone EH, Loop FD et al. (1999): Two internal thoracic artery grafts are better than one. J Thorac Cardiovasc Surg., 117:855-872.

4. Mastrobuoni S, Gawad N. Price J et al. (2012): Use of bilateral internal thoracic artery during coronary artery bypass graft surgery in Canada: The bilateral internal thoracic artery survey. J Thorac Cardiovasc Surg., 144:874-879.

5. Sajja LR (2015): Strategies to reduce deep sternal wound infection after bilateral internal mammary artery grafting. Int J Surg.,16(Pt B):171-178.

6. Grauhan O, Navasardyan A, Tutkun B et al. (2014): Effect of surgical incision management on wound infections in a post sternotomy patient population. Int Wound J., 11:6-9.

7. Mangram Aj, Horan TC, Pearson ML, Silver LC, Jarvis WR (1999): Guideline for prevention of surgical site infection. Hospital Infection Control Practices Advisory Committee. Infect Control Hosp Epidemiol., 20:250-278.

8. Nashef SA, Roques F, Sharples LD et al. (2012): EuroSCORE II. Eur J Cardiothorac Surg., 41:734-744.

9. Online STS Adult Cardiac Surgery Risk Calculator. http://riskcalc.sts.org/stswebriskcalc/\#/ .

10. Atkins BZ, Wooten MK, Kistler J, Hurley K, Hughes GC, Wolfe WG (2009): Does negative pressure wound therapy have a role in preventing poststernotomy wound complications?. Surg Innov., 16:140-146.

11. Colli A, Camara ML (2011): First experience with a new negative pressure incision management system on surgical incisions after cardiac surgery in high risk patients. J Cardiothorac Surg., 6:160.

12. Deo SV, Altarabsheh SE, Shah IK et al. (2015): Are two really always better than one? Results, concerns and controversies in the use of bilateral internal thoracic arteries for coronary artery bypass grafting in the elderly: a systematic review and meta-analysis. Int $\mathbf{J}$ Surg., 16(Pt B):163-70.

13. Grauhan O, Navasardyan A, Tutkun B et al. (2014): Effect of surgical incision management on wound infections in a poststernotomy patient population. Int Wound J., 11:6-9.

14. Grauhan O, Navasardyan A, Hofmann M, Müller P, Stein J (2012): Prevention of poststernotomy wound infections in obese patients by negative pressure wound therapy. J Thorac Cardiovasc Surg., 203; 145:1387-92.

15. O'Leary DP, Peirce C, Anglim B et al. (2017): Prophylactic negative pressure dressing use in closed laparotomy wounds following abdominal operations: a randomized, controlled, open-label trial: The P.I.C.O.Trial. Ann Surg., 265:1082-1086.

16. Li PY, Yang D, Liu D,Sun SJ, Zhang LY (2017): Reducing surgical site infection with negative-pressure wound therapy after open abdominal surgery: a prospective randomized controlled study. Scand J Surg., 106; 3: 189-195.

17. Shen P, Blackham AU, Lewis S et al. (2017): Phase II randomized trial of negative pressure wound therapy to decrease surgical site infection in patients undergoing laparotomy for gastrointestinal, pancreatic, and peritoneal surface malignancies. J Am Coll Surg., 224:726-737.

18. Manoharan V, Grant AL, Harris AC, Hazratwala K, Wilkinson MP, McEwen PJ (2016): Closed incision negative pressure wound therapy vs conventional dry dressings after primary knee arthroplasty: a randomized controlled study. J Arthroplasty, 31: 2487-2494.

19. Masden D, Goldstein J, Endara M, Xu K, Steinberg J, Attinger C (2012): Negative pressure wound therapy for at-risk surgical closures in patients with multiple comorbidities: a prospective randomized controlled study. Ann Surg., 255: 1043-1047. 